Pacific Journal of Mathematics

A THEOREM ON HOLOMORPHIC EXTENSION O 


\title{
A THEOREM ON HOLOMORPHIC EXTENSION OF CR-FUNCTIONS
}

\author{
Guido LuPacciolu
}

\begin{abstract}
We prove the holomorphic extendabilty on a domain $D \in \mathrm{C}^{n}$, $n \geq 2$, of the continuous CR-functions on a relatively open connected subset of $\partial D$, provided the complementary subset of $\partial D$ is $\mathcal{O}(\bar{D})$-convex.
\end{abstract}

Introduction. Let $D$ be a relatively compact open domain in $\mathbf{C}^{n}$, $n \geq 2$, with boundary $\partial D$, and $K$ a compact subset of $\partial D$. We require $D$ and $K$ to be such that $\partial D \backslash K$ is a real hypersurface of class $C^{1}$ in $\mathbf{C}^{n} \backslash K$.

The purpose of this paper is to give a sufficient condition on $D$ and $K$ guaranteeing the holomorphic extendability on all of $D$ of the CR-functions on $\partial D \backslash K$. Our theorem, which states the condition, improves and generalizes previous results in this direction obtained in LupaccioluTomassini [6] and in Tomassini [10]. ${ }^{1}$

Let $\mathcal{O}(\bar{D})$ be the algebra of complex-valued functions on $\bar{D}$ each of which is holomorphic on an open neighborhood of $\bar{D}$, and $\hat{K}_{\bar{D}}$ the $\mathcal{O}(\bar{D})$-hull of $K$. i.e.,

$$
\hat{K}_{\bar{D}}=\bigcap_{\varphi \in \mathcal{O}(\bar{D})}\left\{z \in \bar{D} ;|\varphi(z)| \leq \max _{K}|\varphi|\right\} .
$$

Our main result is the following theorem on holomorphic extension of CR-functions.

TheOREM 1. Assume that $K$ is $\mathcal{O}(\bar{D})$-convex, i.e., $\hat{K}_{\bar{D}}=K$, and $\partial D \backslash K$ is connected. Then every continuous $C R$-function $f$ on $\partial D \backslash K$ has $a$ unique extension $F$ continuous on $\bar{D} \backslash K$ and holomorphic on $D$.

A seemingly more general theorem is the following one.

THEOREM 2. Assume that $\partial D \backslash \hat{K}_{\bar{D}}$ is a connected real hypersurface of class $C^{1}$ in $\mathbf{C}^{n} \backslash \hat{K}_{\bar{D}}$. Then every continuous $C R$-function $f$ on $\partial D \backslash \hat{K}_{\bar{D}}$ has a unique extension $F$ continuous on $\bar{D} \backslash \hat{K}_{\bar{D}}$ and holomorphic on $D \backslash \hat{K}_{\bar{D}}$.

\footnotetext{
${ }^{1}$ Added in proof. Recently Edgar Lee Stout kindly informed me of his paper [12], where the same condition is already recognized to be sufficient, when $D$ is a domain of holomorphy, for a parallel extendability's property in the setting of holomorphic functions.
} 
However, if we set $D^{\prime}=D \backslash \hat{K}_{\bar{D}}$ and $K^{\prime}=\bar{D}^{\prime} \cap \hat{K}_{\bar{D}}$, it is an easy matter to see that Theorem 2 is equivalent to Theorem 1 with $D^{\prime}$ and $K^{\prime}$ in place of $D$ and $K$.

Before going into the proof of Theorem 1, let us discuss a nontrivial situation where it applies.

Observe that, since plainly

$$
\hat{K}_{\bar{D}}=\bigcap_{U \supset \bar{D}} \hat{K}_{U}
$$

where $U$ ranges over the open neighbourhoods of $\bar{D}$, it suffices, in order that $\hat{K}_{\bar{D}}=K$, that, for some $U, \hat{K}_{U} \cap \bar{D}=K$, i.e. $\hat{K}_{U}$ does not meet $\bar{D} \backslash K$. Suppose, then, that the following holds: there is an upper semicontinuous plurisubharmonic function $\rho$ on a Stein open neighbourhood $U$ of $\bar{D}$, so that $K \subset\{\rho=0\}$ and $\bar{D} \backslash K \subset\{\rho>0\}$. Since $\hat{K}_{U}$ coincides with $\hat{K}_{U}^{P}$, the hull of $K$ with respect to the plurisubharmonic functions on $U$ (cf. Hörmander [5], p. 91), it follows that $\hat{K}_{U}$ is contained in $\{\rho \leq 0\}$, and hence $\hat{K}_{U} \cap \bar{D}=K$. In the case $\rho$ is pluriharmonic, $U$ may be required to be simply connected, instead that Stein; for $\rho$ has then a unique pluriharmonic extension $\tilde{\rho}$ to the envelope of holomorphy $\tilde{U}$ of $U$, and hence $\hat{K}_{U} \subset \hat{K}_{\tilde{U}}=\hat{K}_{\tilde{U}}^{P} \subset\{\tilde{\rho} \leq 0\}$.

1. Preliminary facts. (a) We denote by $\omega(\zeta)$ the Martinelli form relative to a point $\zeta=\left(\zeta_{1}, \ldots, \zeta_{n}\right) \in \mathbf{C}^{n}$, that is

$$
\begin{aligned}
\omega(\zeta)= & C_{n} \frac{d z_{1} \wedge \cdots \wedge d z_{n}}{|z-\zeta|^{2 n}} \\
& \wedge \sum_{\alpha=1}^{n}(-1)^{\alpha-1}\left(\bar{z}_{\alpha}-\bar{\zeta}_{\alpha}\right) d \bar{z}_{1} \wedge \cdots \hat{\alpha} \cdots \wedge d \bar{z}_{n}
\end{aligned}
$$

(where $\left.C_{n}=(-1)^{n(n-1) / 2}(n-1) ! /(2 \pi i)^{n}\right)$.

Given a holomorphic function $\varphi$ on an open set $U \subset \mathbf{C}^{n}$ and a point $\zeta \in U$, we denote by $L_{\zeta}(\varphi)$ the level set of $\varphi$ through $\zeta$, that is

$$
L_{\zeta}(\varphi)=\{z \in U ; \varphi(z)=\varphi(\zeta)\} .
$$

It is known that for any $\varphi \in \mathcal{O}(U)$ there exist holomorphic maps $h=$ $\left(h_{1}, \ldots, h_{n}\right) \in \mathcal{O}^{n}(U \times U)$ such that, for each $(z, \zeta) \in U \times U$,

$$
\varphi(z)-\varphi(\zeta)=\sum_{\alpha=1}^{n} h_{\alpha}(z, \zeta)\left(z_{\alpha}-\zeta_{\alpha}\right)
$$

(cf. Harvey [3], Lemma 2.3). Then we set:

$$
\mathcal{O}_{\varphi}^{n}(U \times U)=\left\{h \in \mathcal{O}^{n}(U \times U) ;(*) \text { holds }\right\} .
$$

Any $h \in \mathcal{O}_{\varphi}^{n}(U \times U)$ allows one to define canonically, for $\zeta \in U$, a $\bar{\partial}$-primitive of $\omega(\zeta)$ on $U \backslash L_{\zeta}(\varphi)$, that is $(n, n-2)$-form $\Phi_{h}(\zeta)$ on 
$U \backslash L_{\zeta}(\varphi)$ such that

$$
\omega(\zeta)=\bar{\partial} \Phi_{h}(\zeta)=d \Phi_{h}(\zeta)
$$

As a matter of fact, consider, for every $\alpha=1, \ldots, n$, the following $(n, n-2)$-form on $\mathbf{C}^{n} \backslash\left\{z_{\alpha}=\zeta_{\alpha}\right\}=\mathbf{C}^{n} \backslash L_{\zeta}\left(z_{\alpha}\right)$ :

$$
\begin{aligned}
\Omega_{\alpha}(\zeta)= & \frac{(-1)^{n+\alpha}}{n-1} C_{n} \frac{d z_{1} \wedge \cdots \wedge d z_{n}}{\left(z_{\alpha}-\zeta_{\alpha}\right)|z-\zeta|^{2 n-2}} \\
& \wedge\left[\sum_{\beta=1}^{\alpha-1}(-1)^{\beta}\left(\bar{z}_{\beta}-\bar{\zeta}_{\beta}\right) d \bar{z}_{1} \wedge \cdots \hat{\beta} \cdots \hat{\alpha} \cdots \wedge d \bar{z}_{n}\right. \\
& \left.\quad+\sum_{\beta=\alpha+1}^{n}(-1)^{\beta-1}\left(\bar{z}_{\beta}-\bar{\zeta}_{\beta}\right) d \bar{z}_{1} \wedge \cdots \hat{\alpha} \cdots \hat{\beta} \cdots \wedge d \bar{z}_{n}\right] .
\end{aligned}
$$

One verifies that, on $\mathbf{C}^{n} \backslash L_{\zeta}\left(z_{\alpha}\right), \omega(\zeta)=\bar{\partial} \Omega_{\alpha}(\zeta) .{ }^{2}$ Then set

$$
\Phi_{h}(\zeta)=\frac{1}{\varphi(z)-\varphi(\zeta)} \sum_{\alpha=1}^{n} h_{\alpha}(z, \zeta)\left(z_{\alpha}-\zeta_{\alpha}\right) \Omega_{\alpha}(\zeta)
$$

It is plain that $\Phi_{h}(\zeta)$ is indeed a real analytic $\bar{\partial}$-primitive of $\omega(\zeta)$ on $U \backslash L_{\zeta}(\varphi)$.

Such $\bar{\partial}$-primitives of the Martinelli form will play a fundamental role in the proof of our extension theorem. Now we derive the properties of them that will be needed.

Let there be given open sets $U, U^{\prime} \subset C^{n}$ such that $U \cap U^{\prime} \neq$ $\varnothing$, functions $\varphi \in \mathcal{O}(U), \varphi^{\prime} \in \mathcal{O}\left(U^{\prime}\right)$ and maps $h \in \mathcal{O}_{\varphi}^{n}(U \times U), h^{\prime} \in$ $\mathcal{O}_{\varphi^{\prime}}^{n}\left(U^{\prime} \times U^{\prime}\right)$, and let $\zeta$ be a point in $U \cap U^{\prime}$. Suppose first that $n \geq 3$, and consider, for every $\alpha, \beta=1, \ldots, n$ with $\alpha \neq \beta$, the $(n, n-3)$ form $\Lambda_{\alpha, \beta}(\zeta)$ on $\mathbf{C}^{n} \backslash\left(L_{\zeta}\left(z_{\alpha}\right) \cup L_{\zeta}\left(z_{\beta}\right)\right)$ defined as follows: for $\alpha<\beta$

$$
\begin{aligned}
\Lambda_{\alpha, \beta}(\zeta) & =\frac{(-1)^{n+\alpha+\beta}}{(n-1)(n-2)} C_{n} \frac{d z_{1} \wedge \cdots \wedge d z_{n}}{\left(z_{\alpha}-\zeta_{\alpha}\right)\left(z_{\beta}-\zeta_{\beta}\right)|z-\zeta|^{2 n-4}} \\
\wedge & {\left[\sum_{\gamma=1}^{\alpha-1}(-1)^{\gamma}\left(\bar{z}_{\gamma}-\bar{\zeta}_{\gamma}\right) d \bar{z}_{1} \wedge \cdots \hat{\gamma} \cdots \hat{\alpha} \cdots \hat{\beta} \cdots \wedge d \bar{z}_{n}\right.} \\
& +\sum_{\gamma=\alpha+1}^{\beta-1}(-1)^{\gamma-1}\left(\bar{z}_{\gamma}-\bar{\zeta}_{\gamma}\right) d \bar{z}_{1} \wedge \cdots \hat{\alpha} \cdots \hat{\gamma} \cdots \hat{\beta} \cdots \wedge d \bar{z}_{n} \\
& \left.+\sum_{\gamma=\beta+1}^{n}(-1)^{\gamma}\left(\bar{z}_{\gamma}-\bar{\zeta}_{\gamma}\right) d \bar{z}_{1} \wedge \cdots \hat{\alpha} \cdots \hat{\beta} \cdots \hat{\gamma} \cdots \wedge d \bar{z}_{n}\right],
\end{aligned}
$$

${ }^{2}$ The forms $\Omega_{\alpha}(\zeta)$ were considered first by Martinelli [7], to give a proof of Hartogs' theorem. 
and for $\alpha>\beta \Lambda_{\alpha, \beta}(\zeta)=-\Lambda_{\beta, \alpha}(\zeta)$. One can verify that $\Omega_{\alpha}(\zeta)-\Omega_{\beta}(\zeta)=$ $\bar{\partial} \Lambda_{\alpha, \beta}(\zeta)$. Then, consider the following $(n, n-3)$-form on $\left(U \backslash L_{\zeta}(\varphi)\right) \cap$ $\left(U^{\prime} \backslash L_{\zeta}\left(\varphi^{\prime}\right)\right)$ :

$$
\begin{aligned}
\mathrm{X}_{h, h^{\prime}}(\zeta)= & \frac{1}{(\varphi(z)-\varphi(\zeta))\left(\varphi^{\prime}(z)-\varphi^{\prime}(\zeta)\right)} \\
& \cdot \sum_{1 \leq \alpha<\beta \leq n}\left(h_{\alpha} h_{\beta}^{\prime}-h_{\beta} h_{\alpha}^{\prime}\right)\left(z_{\alpha}-\zeta_{\alpha}\right)\left(z_{\beta}-\zeta_{\beta}\right) \Lambda_{\alpha, \beta}(\zeta) .
\end{aligned}
$$

It is easily seen that, on $\left(U \backslash L_{\zeta}(\varphi)\right) \cap\left(U^{\prime} \backslash L_{\zeta}\left(\varphi^{\prime}\right)\right)$,

$$
\Phi_{h}(\zeta)-\Phi_{h^{\prime}}(\zeta)=\bar{\partial} X_{h, h^{\prime}}(\zeta)
$$

In case $n=2$ we simply have:

$$
\Omega_{1}(\zeta)-\Omega_{2}(\zeta)=-\frac{1}{(2 \pi i)^{2}} \frac{d z_{1} \wedge d z_{2}}{\left(z_{1}-\zeta_{1}\right)\left(z_{2}-\zeta_{2}\right)},
$$

and hence we find, on $\left(U \backslash L_{\zeta}(\varphi)\right) \cap\left(U^{\prime} \backslash L_{\zeta}\left(\varphi^{\prime}\right)\right)$ :

(1.4) $\Phi_{h}(\zeta)-\Phi_{h^{\prime}}(\zeta)=-\frac{1}{(2 \pi i)^{2}} \frac{\left(h_{1} h_{2}^{\prime}-h_{2} h_{1}^{\prime}\right) d z_{1} \wedge d z_{2}}{(\varphi(z)-\varphi(\zeta))\left(\varphi^{\prime}(z)-\varphi^{\prime}(\zeta)\right)}$.

Next, we observe that all the above differential forms depend in a real analytic fashion also on the point $\zeta$, so that we may perform any derivative of these with respect to the parameters $\operatorname{Re} \zeta_{\alpha}, \operatorname{Im} \zeta_{\alpha}, \alpha=1, \ldots, n$ (by taking the derivative of each coefficient). In particular we may consider the forms $\partial \omega / \partial \bar{\xi}_{\alpha}, \partial \Omega_{\beta} / \partial \bar{\xi}_{\alpha}$, etc., obtained by applying the Wirtinger operator $\partial \cdot / \partial \bar{\zeta}_{\alpha}$. We first note that, for every $\alpha=1, \ldots, n$, the $(n, n-2)$-form $\partial \Omega_{\alpha} / \partial \bar{\zeta}_{\alpha}$ satisfies

$$
\frac{\partial \Omega_{\alpha}}{\partial \bar{\zeta}_{\alpha}}(\zeta)=(n-1) \frac{z_{\alpha}-\zeta_{\alpha}}{|z-\zeta|^{2}} \Omega_{\alpha}(\zeta)
$$

and hence is defined (and real analytic) on $\mathbf{C}^{n} \backslash \zeta$, instead that only on $\mathbf{C}^{n} \backslash L_{\zeta}\left(z_{\alpha}\right)$ as $\Omega_{\alpha}(\zeta)$. It follows that, on $\mathbf{C}^{n} \backslash \zeta$,

$$
\frac{\partial \omega}{\partial \bar{\zeta}_{\alpha}}(\zeta)=\bar{\partial}\left[\frac{\partial \Omega_{\alpha}}{\partial \bar{\zeta}_{\alpha}}(\zeta)\right] \quad(\alpha=1, \ldots, n) .
$$

Similarly, if $n \geq 3$, for every $\alpha, \beta=1, \ldots, n$ with $\alpha \neq \beta$, the $(n, n-3)$ form $\partial \Lambda_{\alpha, \beta} / \partial \bar{\zeta}_{\alpha}$ satisfies

$$
\frac{\partial \Lambda_{\alpha, \beta}}{\partial \bar{\zeta}_{\alpha}}(\zeta)=(n-2) \frac{z_{\alpha}-\zeta_{\alpha}}{|z-\zeta|^{2}} \Lambda_{\alpha, \beta}(\zeta)
$$


and hence is defined on $\mathbf{C}^{n} \backslash L_{\zeta}\left(z_{\beta}\right)$, instead that only on $\mathbf{C}^{n} \backslash\left(L_{\zeta}\left(z_{\alpha}\right) \cup\right.$ $\left.L_{\zeta}\left(z_{\beta}\right)\right)$ as $\Lambda_{\alpha, \beta}(\zeta)$. It follows that, on $\mathbf{C}^{n} \backslash L_{\zeta}\left(z_{\beta}\right)$,

$$
\frac{\partial \Omega_{\alpha}}{\partial \bar{\zeta}_{\alpha}}(\zeta)-\frac{\partial \Omega_{\beta}}{\partial \bar{\zeta}_{\alpha}}(\zeta)=\bar{\partial}\left[\frac{\partial \Lambda_{\alpha, \beta}}{\partial \bar{\zeta}_{\alpha}}(\zeta)\right] .
$$

If $n=2$ we simply have, for $\alpha=1,2$ :

$$
\frac{\partial \Omega_{1}}{\partial \bar{\zeta}_{\alpha}}(\zeta)-\frac{\partial \Omega_{2}}{\partial \bar{\zeta}_{\alpha}}(\zeta)=0
$$

Now, let there be given an open set $U \subset \mathbf{C}^{n}$, a function $\varphi \in \mathcal{O}(U)$ and a map $h \in \mathcal{O}_{\varphi}^{n}(U \times U)$, and let $\zeta$ be a point in $U$. In case $n \geq 3$ consider, for every $\alpha=1, \ldots, n$, the following ( $n, n-3)$-form on $U \backslash L_{\zeta}(\varphi)$ :

$$
\Psi_{h}^{\alpha}(\zeta)=\frac{1}{\varphi(z)-\varphi(\zeta)} \sum_{\substack{\beta=1 \\ \beta \neq \alpha}}^{n} h_{\beta}\left(z_{\beta}-\zeta_{\beta}\right) \frac{\partial \Lambda_{\alpha, \beta}}{\partial \bar{\zeta}_{\alpha}}(\zeta) .
$$

Then we find, on $U \backslash L_{\zeta}(\varphi)$ :

$$
\frac{\partial \Phi_{h}}{\partial \bar{\zeta}_{\alpha}}(\zeta)=\frac{\partial \Omega_{\alpha}}{\partial \bar{\zeta}_{\alpha}}(\zeta)-\bar{\partial} \Psi_{h}^{\alpha}(\zeta) \quad(\alpha=1, \ldots, n)
$$

On the other hand, if $n=2$, we have:

$$
\frac{\partial \Phi_{h}}{\partial \bar{\zeta}_{\alpha}}(\zeta)=\frac{\partial \Omega_{\alpha}}{\partial \bar{\zeta}_{\alpha}}(\zeta) \quad(\alpha=1,2)
$$

(b) It is well known that, given an oriented real hypersurface $\Sigma$ of class $C^{1}$ in $\mathbf{C}^{n}$ (without boundary, not necessarily closed) and a complexvalued function $f$ in $L_{\text {loc }}^{1}(\Sigma)$, one may say that $f$ is a CR-function on $\Sigma$ in case it satisfies the tangential Cauchy-Riemann equation in the weak form, that is

$$
\int_{\Sigma} f \bar{\partial} \lambda=0
$$

for every $(n, n-2)$-form $\lambda$ of class $C^{1}$ on an open neighbourhood of $\Sigma$, such that $\Sigma \cap \operatorname{Supp}(\lambda)$ is compact. However we need for our purposes a sharper characterization of continuous CR-functions on $\Sigma$ than (1.8) is. This is provided by the following proposition.

Proposition 1.9. Let $f$ be a complex-valued continuous function on $\Sigma$. Then $f$ is a CR-function if and only if it satisfies

$$
\int_{c_{n+q}} f \bar{\partial} \mu=\int_{\partial c_{n+q}} f \mu,
$$


for every singular $(n+q)$-chain $c_{n+q}$ of $\Sigma$ of class $C^{1}$ and every $(n, q-1)$ form $\mu$ of class $C^{1}$ on an open neighbourhood of $\Sigma(1 \leq q \leq n-1) .^{3}$

Proof. This proposition asserts that (1.8) and (1.10) are equivalent for a continuous $f$ (which would be quite immediate if $f$ were of class $C^{1}$ ). We shall prove only that (1.8) implies (1.10), the converse being trivial.

For every differential form $\mu$ of class $C^{1}$ on an open neighbourhood $V$ of $\Sigma$, we denote by $\left.\mu\right|_{\Sigma}$ the restriction of $\mu$ to $\Sigma$ (i.e. the pull-back of $\mu$ by the inclusion map $\Sigma \hookrightarrow V$ ). Then $\left.\mu\right|_{\Sigma}$ is a continuous regular form on $\sum{ }^{4}$

Consider the continuous $n$-form on $\Sigma$

$$
u=\left.f\left(d z_{1} \wedge \cdots \wedge d z_{n}\right)\right|_{\Sigma} .
$$

We claim that (1.10) is equivalent to the following assertion:

$u$ is regular on $\Sigma$ and $d u=0$.

As a matter of fact, taking in particular $q=1$ and $\mu=d z_{1} \wedge \cdots \wedge d z_{n}$, (1.10) gives:

$$
0=\int_{\partial c_{n+1}} f d z_{1} \wedge \cdots \wedge d z_{n}=\int_{\partial c_{n+1}} u
$$

for every singular $(n+1)$-chain $c_{n+1}$ of $\Sigma$ of class $C^{1}$; and this is just as to say that $(*)$ holds. Conversely, assume that $(*)$ holds. Any $(n, q-1)$ form $\mu$ as in the statement can be written as $\mu=d z_{1} \wedge \cdots \wedge d z_{n} \wedge \tilde{\mu}$, where $\tilde{\mu}$ is a $(0, q-1)$-form of class $C^{1}$ on an open neighbourhood of $\Sigma$. Then $\left.u \wedge \tilde{\mu}\right|_{\Sigma}$ is a continuous regular $(n+\mathrm{q}-1)$-form on $\Sigma$ and, since $d u=0, d\left(\left.\tilde{\mu}\right|_{\Sigma}\right)=\left.(d \tilde{\mu})\right|_{\Sigma}$, we have:

$$
d\left(\left.u \wedge \tilde{\mu}\right|_{\Sigma}\right)=\left.(-1)^{n} u \wedge(d \tilde{\mu})\right|_{\Sigma}=\left.f(d \mu)\right|_{\Sigma}=\left.f(\bar{\partial} \mu)\right|_{\Sigma} .
$$

It follows that

$$
\int_{c_{n+q}} f \bar{\partial} \mu=\left.\int_{\partial c_{n+q}} u \wedge \tilde{\mu}\right|_{\Sigma}=\int_{\partial c_{n+q}} f \mu
$$

that is, (1.1) holds. Next, we claim that $(*)$ is equivalent to:

$(* *) \quad u$ is weakly closed on $\Sigma$, that is $\int_{\Sigma} u \wedge d v=0$

for every $(n-2)$-form $v$ on $\Sigma$ of class $C^{1}$ and with compact support.

\footnotetext{
${ }^{3}$ The same result is proved in Lupacciolu-Tomassini [6] under the additional assumption that $f$ is locally Lipschitz, but the argument used there does not work without that assumption.

${ }^{4}$ For the definition and basic properties of continuous regular forms we refer to Whitney [11] pp. 103-108. We denote, as usual, by $d$ the differential acting on such forms (defined by means of Stokes' formula), as the ordinary exterior differential.
} 
This latter equivalence is a straightforward consequence of the following general facts about continuous differential forms on a manifold of class $C^{1}$ :

(i) The differential acting on continuous regular forms may be understood in the strong sense. This means that, if $\eta, \theta$ are continuous forms, then $\eta, \theta$ are regular and $d \eta=\theta$ in the sense of regular forms if and only if there exists a sequence $\left\{\eta_{s}\right\}_{s=1}^{\infty}$ of forms of class $C^{1}$ such that $\eta_{s} \rightarrow \eta$ and $d \eta_{s} \rightarrow \theta$ as $s \rightarrow \infty$, both uniformly on compact sets (cf. Whitney [11]);

(ii) The differential in the strong sense coincides with the differential in the weak sense. This means that, if $\eta, \theta$ are continuous forms, then $d \eta=\theta$ in the strong sense if and only if $\int \eta \wedge d \xi=(-1)^{\operatorname{deg} \eta+1} \int \theta \wedge \xi$, for every form $\xi$ of class $C^{1}$ and with compact support (cf. Friedrichs [2], or Fichera [1]). ${ }^{5}$

Now we show that $(1.8)$ implies $(* *)$, which will conclude the proof. We shall use the following fact: there exists an open neighbourhood $W$ of $\Sigma$ in $\mathbf{C}^{n}$ and a retraction $r: W \rightarrow \Sigma$ of class $C^{1}$ (which means that $r(z)=z$ for each $z \in \Sigma$ ). This is a special case of a standard theorem in Differential Topology (cf. Munkres [8], p. 51, or Whitney [11], p. 121). ${ }^{6}$ If $v$ is any $(n-2)$-form on $\Sigma$ of class $C^{1}$ and with compact support, consider its pull-back $r^{*} v$ to $W . r^{*} v$ is a continuous regular $(n-2)$-form on $W$, and hence we can find a sequence $\left\{\eta_{s}\right\}_{s=1}^{\infty}$ of $(n-2)$-forms of class $C^{1}$ on $W$ such that

$$
\lim _{s \rightarrow \infty} \eta_{s}=r^{*} v, \quad \lim _{s \rightarrow \infty} d \eta_{s}=r^{*} d v,
$$

both uniformly on compact subsets of $W$. Moreover, since $\Sigma \cap$ $\operatorname{Supp}\left(r^{*} v\right)=\operatorname{Supp}(v)$ is compact, we can arrange that so too is $\Sigma \cap$ $\operatorname{Supp}\left(\eta_{s}\right)$, for every $s$. It follows that

$$
\begin{aligned}
\int_{\Sigma} u \wedge d v & =\left.\lim _{s \rightarrow \infty} \int_{\Sigma} u \wedge\left(d \eta_{s}\right)\right|_{\Sigma} \\
& =\lim _{s \rightarrow \infty} \int_{\Sigma} f d z_{1} \wedge \cdots \wedge d z_{n} \wedge d \eta_{s} \\
& =(-1)^{n} \lim _{s \rightarrow \infty} \int_{\Sigma} f \bar{\partial}\left(d z_{1} \wedge \cdots \wedge d z_{n} \wedge \eta_{s}\right),
\end{aligned}
$$

and hence (1.8) implies $\int_{\Sigma} u \wedge d v=0$.

\footnotetext{
${ }^{5}$ Clearly, the interest of this fact is in the "if", the "only if" being trivial.

${ }^{6}$ If $\Sigma$ were of class $C^{2}$, we could use the more elementary "tubular neighbourhood theorem".
} 
2. Proof of Theorem 1. Let $V$ be an open neighbourhood of $K$ in $\mathbf{C}^{n}$ and $\sigma: \mathbf{C}^{n} \rightarrow \mathbf{R}$ a $C^{\infty}$ function such that $0 \leq \sigma(z) \leq 1$ for all $z$, $\sigma(z)=1$ for $z \in K, \operatorname{Supp}(\sigma)$ is compact and contained in $V$. For a generic small $\varepsilon>0$, set $D_{\varepsilon}=D \cap\{1-\sigma>\varepsilon\}, \Gamma_{\varepsilon}=\partial D \cap\{1-\sigma \geq \varepsilon\}$ and $K_{\varepsilon}=\bar{D} \cap\{1-\sigma=\varepsilon\}$. Then $D_{\varepsilon}$ is a subdomain of $D, \partial D_{\varepsilon}=\Gamma_{\varepsilon} \cup$ $K_{\varepsilon}, \Gamma_{\varepsilon}$ and $K_{\varepsilon}$ are compact real hypersurfaces with boundary, of class $C^{1}$, such that $\Gamma_{\varepsilon} \cap K_{\varepsilon}=\partial \Gamma_{\varepsilon}=\partial K_{\varepsilon}$, and $\Gamma_{\varepsilon}$ is connected. Clearly, $D$ is exhaustible by an increasing sequence of subdomains of this sort, $\left\{D_{s}\right\}_{s=1}^{\infty}$, say, so that

$$
\partial D_{s}=\Gamma_{s} \cup K_{s} \quad(s=1,2, \ldots),
$$

with obvious meaning of $\Gamma_{s}, K_{s}$, and

$$
D=\bigcup_{s=1}^{\infty} D_{s}, \quad \partial D \backslash K=\bigcup_{s=1}^{\infty} \Gamma_{s} .
$$

We assume that the sequence $\left\{D_{s}\right\}_{s=1}^{\infty}$ has been chosen once for all.

Now, let $U$ be an open neighbourhood of $\bar{D}$ and let $\varphi \in \mathcal{O}(U)$. For every positive integer $s$ we set:

$$
U_{s}(\varphi)=\left\{\zeta \in U ;|\varphi(\zeta)|>\frac{\max }{D \backslash D_{s}}|\varphi|\right\} .
$$

Then $U_{s}(\varphi)$ is an open subset of $U \backslash \overline{D \backslash D_{s}}$ such that, if $\zeta \in U_{s}(\varphi)$, the level set $L_{\zeta}(\varphi)$ of $\varphi$ through $\zeta$ is all contained in $U_{s}(\varphi)$. Moreover we set:

$$
U(\varphi)=\left\{\zeta \in U ;|\varphi(\zeta)|>\max _{K}|\varphi|\right\} .
$$

Since $\left\{\overline{D \backslash D_{s}}\right\}_{s=1}^{\infty}$ is a decreasing sequence of compact neighbourhoods of $K$ in $\bar{D}$ such that $K=\bigcap_{s=1}^{\infty} \overline{D \backslash D_{s}}$, it follows that $U_{1}(\varphi) \subset U_{2}(\varphi) \cdots$, and

$$
U(\varphi)=\bigcup_{s=1}^{\infty} U_{s}(\varphi) .
$$

Moreover, since $\hat{K}_{\bar{D}}=\bigcap_{U \supset \bar{D}} \hat{K}_{U}$ (where $U$ ranges over the open neighbourhoods of $\bar{D}$ ), the assumption of Theorem 1 implies:

$$
\bar{D} \backslash K \subset \bigcup_{U \supset \bar{D}} \bigcup_{\varphi \in \mathcal{O}(U)} U(\varphi) .
$$

Next, for every $U, \varphi, s$ as above and $h \in \mathcal{O}_{\varphi}^{n}(U \times U)$ (cf. (1.1)), consider the complex-valued function $F_{h}^{s}$ on $U_{s}(\varphi) \backslash \partial D$ given by

$$
F_{h}^{s}(\zeta)=\int_{\Gamma_{s}} f \omega(\zeta)-\int_{\partial \Gamma_{s}} f \Phi_{h}(\zeta)
$$


where $\Phi_{h}(\zeta)$ is the $\bar{\partial}$-primitive (1.2) of the Martinelli form $\omega(\zeta), \Gamma_{s}$ is oriented as a part of $\partial D$ and $\partial \Gamma_{s}$ as the boundary of $\Gamma_{s}{ }^{7}$ Since, for $\zeta \in U_{s}(\varphi)$ and $z \in \partial \Gamma_{s},|\varphi(\zeta)|>|\varphi(z)|$ (because $\partial \Gamma_{s} \subset \overline{D \backslash D_{s}}$ ), the singular set $L_{\zeta}(\varphi)$ of $\Phi_{h}(\zeta)$ does not meet $\partial \Gamma_{s}$, so that $F_{h}^{s}$ is indeed defined, and real analytic, on $U_{s}(\varphi) \backslash \Gamma_{s}=U_{s}(\varphi) \backslash \partial D$.

Proposition 2.4. Suppose there exists at least a function $F$ as in the statement of Theorem 1. Then, for every $U, \varphi, h, s$ as above,

$$
F=F_{h}^{s} \text { on } D \cap U_{s}(\varphi) \text {. }
$$

As a consequence, on account of (2.1) and (2.2), if such a $F$ actually exists, it is necessarily unique.

Proof. Clearly $D \cap U_{s}(\varphi) \subset D_{s}$, and, by assumption, $F \in C^{0}\left(\bar{D}_{s}\right) \cap$ $\mathcal{O}\left(D_{s}\right)$ and $F=f$ on $\Gamma_{s}$. Therefore, since, by the Martinelli formula, for $\zeta \in D_{s}$, we have:

$$
F(\zeta)=\int_{\Gamma_{s}} f \omega(\zeta)+\int_{K_{s}} F \omega(\zeta),
$$

we are required to show that, for $\zeta \in D \cap U_{s}(\varphi)$, we also have:

$$
\int_{K_{s}} F \omega(\zeta)=-\int_{\partial \Gamma_{s}} f \Phi_{h}(\zeta)
$$

Since $F$ is continuous on $\bar{D} \backslash K$ and holomorphic on $D$, the forms $F \omega(\zeta)$, $F \Phi_{h}(\zeta)$ are both continuous on $(\bar{D} \backslash K) \backslash L_{\zeta}(\varphi)$, real analytic on $D \backslash L_{\zeta}(\varphi)$, and on $D \backslash L_{\zeta}(\varphi)$ satisfy $F \omega(\zeta)=d\left(F \Phi_{h}(\zeta)\right)$. Moreover, since $\zeta \in U_{s}(\varphi)$, it follows that $K_{s} \subset(\bar{D} \backslash K) \backslash L_{\zeta}(\varphi)$. Then consider the restrictions $\left.(F \omega(\zeta))\right|_{K_{s}},\left.\left(F \Phi_{h}(\zeta)\right)\right|_{K_{s}}$; these are continuous on $K_{s}$, regular on $K_{s} \backslash \partial K_{s}$ and on $K_{s} \backslash \partial K_{s}$ satisfy $\left.(F \omega(\zeta))\right|_{K_{s}}=d\left[\left.\left(F \Phi_{h}(\zeta)\right)\right|_{K_{s}}\right]$. Hence Stokes' theorem for regular forms on a manifold with boundary (cf. Whitney [11], p. 109) implies:

$$
\int_{K_{s}} F \omega(\zeta)=\int_{\partial K_{s}} F \Phi_{n}(\zeta)
$$

Finally, since $\partial K_{s}=-\partial \Gamma_{s}\left(=\partial \Gamma_{s}\right.$ with the opposite orientation), $(*)$ follows.

The above proposition disposes of the uniqueness' assertion in Theorem 1 and, further, implies that the proof of the existence of a holomor-

\footnotetext{
${ }^{7}$ In this paper we take as the canonical orientation of $\mathbf{C}^{n}$ and of $D$ the one given by the volume-form $(i / 2)^{n} d z_{1} \wedge d \bar{z}_{1} \wedge \cdots \wedge d z_{n} \wedge d \bar{z}_{n}$.
} 
phic continuation of $f$ on $D$ shall be a matter of showing that the $F_{h}^{s}$ 's do in fact define a holomorphic function $F$ on $D$ such that, for each $z^{0} \in \partial D \backslash K, F(\zeta) \rightarrow f\left(z^{0}\right)$ as $\zeta \rightarrow z^{0}$ in $D$. In the first place we have:

Proposition 2.5. The functions $F_{h}^{s^{\prime}}$ s are each other coherent and holomorphic. Hence there is a unique holomorphic function $F$ on

$$
\left(\bigcup_{U \supset \bar{D}} \bigcup_{\varphi \in \mathcal{O}(U)} U(\varphi)\right) \backslash \partial D
$$

such that, for every $U, \varphi, h, s$,

$$
F=F_{h}^{s} \text { on } U_{s}(\varphi) \backslash \partial D .
$$

Proof. We first prove the coherence. This means that, for every $U, \varphi, h, s$ and $U^{\prime}, \varphi^{\prime}, h^{\prime}, s^{\prime}$, we have:

$$
F_{h}^{s}=F_{h^{\prime}}^{s^{\prime}} \quad \text { on } U_{s}(\varphi) \cap U_{s^{\prime}}^{\prime}\left(\varphi^{\prime}\right) \backslash \partial D .
$$

We may assume that $s \geq s^{\prime}$. Then $(*)$ will be a consequence of the following two equalities:

(i) $F_{h^{\prime}}^{s}=F_{h^{\prime}}^{s^{\prime}}$ on $U_{s^{\prime}}^{\prime}\left(\varphi^{\prime}\right) \backslash \partial D$;

(ii) $F_{h}^{s}=F_{h^{\prime}}^{s}$ on $U_{s}(\varphi) \cap U_{s}^{\prime}\left(\varphi^{\prime}\right) \backslash \partial D$

(recall that $U_{s^{\prime}}(\varphi) \subset U_{s}(\varphi)$ and $U_{s^{\prime}}^{\prime}\left(\varphi^{\prime}\right) \subset U_{s}^{\prime}\left(\varphi^{\prime}\right)$ ). To prove (i) (in case $\left.s>s^{\prime}\right)$, consider the $(2 n-1)$-chain of $\partial D \backslash K$, of class $C^{1}, c_{2 n-1}=\Gamma_{s}-$ $\Gamma_{s^{\prime}}$ If $\zeta$ is any point in $U_{s^{\prime}}^{\prime}\left(\varphi^{\prime}\right) \backslash \partial D$, it is plain that

$$
F_{h^{\prime}}^{s}(\zeta)-F_{h^{\prime}}^{s^{\prime}}(\zeta)=\int_{c_{2 n-1}} f \omega(\zeta)-\int_{\partial c_{2 n-1}} f \Phi_{h^{\prime}}(\zeta)
$$

moreover, since $\operatorname{Supp}\left(c_{2 n-1}\right) \subset \overline{D_{s} \backslash D_{s^{\prime}}} \subset \overline{D \backslash D_{s^{\prime}}}$ and $L_{\zeta}\left(\varphi^{\prime}\right) \subset U_{s^{\prime}}^{\prime}\left(\varphi^{\prime}\right)$ $\subset U^{\prime} \backslash \overline{D \backslash D_{s^{\prime}}}$, it follows that $\operatorname{Supp}\left(c_{2 n-1}\right)$ is contained in $U^{\prime} \backslash L_{\zeta}\left(\varphi^{\prime}\right)$, where $\omega(\zeta), \Phi_{h^{\prime}}(\zeta)$ are both defined and satisfy $\omega(\zeta)=\bar{\partial} \Phi_{h^{\prime}}(\zeta)$. Then, if we take a $(n, n-2)$-form $\mu$ of class $C^{\infty}$ on all of $\mathbf{C}^{n}$ and equal to $\Phi_{h^{\prime}}(\zeta)$ on an open neighbourhood of $\operatorname{Supp}\left(c_{2 n-1}\right)$, we may replace $\omega(\zeta), \Phi_{h^{\prime}}(\zeta)$, in the right side of the above equality, respectively by $\bar{\partial} \mu, \mu$. Hence Proposition 1.9 gives at once that $F_{h^{\prime}}^{s}(\zeta)=F_{h^{\prime}}^{s^{\prime}}(\zeta)$.

Next we prove (ii). On account of (1.3), (1.4), we have, for each $\zeta \in U_{s}(\varphi) \cap U_{s}^{\prime}\left(\varphi^{\prime}\right) \backslash \partial D:$

$$
\begin{aligned}
& F_{h}^{s}(\zeta)-F_{h^{\prime}}^{s}(\zeta) \\
& = \begin{cases}-\int_{\partial \Gamma_{s}} f \bar{\partial} X_{h, h^{\prime}}(\zeta) & \text { if } n \geq 3, \\
\frac{1}{(2 \pi i)^{2}} \int_{\partial \Gamma_{s}} f(z) \frac{\left(h_{1} h_{2}^{\prime}-h_{2} h_{1}^{\prime}\right) d z_{1} \wedge d z_{2}}{(\varphi(z)-\varphi(\zeta))\left(\varphi^{\prime}(z)-\varphi^{\prime}(\zeta)\right)} & \text { if } n=2 .\end{cases}
\end{aligned}
$$


In case $n \geq 3$, we may replace $\mathrm{X}_{h, h^{\prime}}(\zeta)$, in the integral on the right side, by any $(n, n-3)$-form $\tilde{X}$ of class $C^{\infty}$ on all of $\mathbf{C}^{n}$ and equal to $\mathrm{X}_{h, h^{\prime}}(\zeta)$ on an open neighbourhood of $\partial \Gamma_{s}$. Hence Proposition 1.9 (for $q=n-1$, $c_{n+q}=\Gamma_{s}$ and $\left.\mu=\bar{\partial} \tilde{X}\right)$ implies that $F_{h}^{s}(\zeta)=F_{h^{\prime}}^{s}(\zeta)$.

In case $n=2$, we have to argue differently. Since $\zeta \in U_{s}(\varphi) \cap U_{s}^{\prime}\left(\varphi^{\prime}\right)$ and $\partial \Gamma_{s} \subset \overline{D \backslash D_{s}}$, it follows that, for each $z \in \partial \Gamma_{s},|\varphi(\zeta)|>\max _{\overline{D \backslash D_{s}}}|\varphi|$ $\geq|\varphi(z)|$, and hence $|\varphi(z) / \varphi(\zeta)|<1$. Similarly, $\left|\varphi^{\prime}(z) / \varphi^{\prime}(\zeta)\right|<1$. Therefore we may write, for $z \in \partial \Gamma_{s}$ :

$$
\begin{aligned}
& \frac{1}{(\varphi(z)-\varphi(\zeta))}\left(\varphi^{\prime}(z)-\varphi^{\prime}(\zeta)\right) \\
& =\frac{1}{\varphi(\zeta) \varphi^{\prime}(\zeta)} \cdot \frac{1}{(1-\varphi(z) / \varphi(\zeta))\left(1-\varphi^{\prime}(z) / \varphi^{\prime}(\zeta)\right)} \\
& =\frac{1}{\varphi(\zeta) \varphi^{\prime}(\zeta)} \sum_{\alpha, \beta}^{0, \infty}\left(\frac{\varphi(z)}{\varphi(\zeta)}\right)^{\alpha}\left(\frac{\varphi^{\prime}(z)}{\varphi^{\prime}(\zeta)}\right)^{\beta},
\end{aligned}
$$

with the double series absolutely uniformly convergent on $\partial \Gamma_{s}$. It follows that

$$
\begin{aligned}
\int_{\partial \Gamma_{s}} f(z) & \frac{\left(h_{1} h_{2}^{\prime}-h_{2} h_{1}^{\prime}\right) d z_{1} \wedge d z_{2}}{(\varphi(z)-\varphi(\zeta))\left(\varphi^{\prime}(z)-\varphi^{\prime}(\zeta)\right)} \\
& =\sum_{\alpha, \beta}^{0, \infty} \frac{1}{(\varphi(\zeta))^{\alpha+1}\left(\varphi^{\prime}(\zeta)\right)^{\beta+1}} \int_{\partial \Gamma_{s}} f \mu_{\alpha, \beta}
\end{aligned}
$$

where

$$
\begin{aligned}
\mu_{\alpha, \beta}=\left(h_{1} h_{2}^{\prime}-h_{2} h_{1}^{\prime}\right)(\varphi(z))^{\alpha}\left(\varphi^{\prime}(z)\right)^{\beta} d z_{1} \wedge d z_{2} \\
(\alpha, \beta=0,1,2, \ldots) .
\end{aligned}
$$

Now, since every $\mu_{\alpha, \beta}$ is a holomorphic 2-form on $U \cap U^{\prime}$, so that $\bar{\partial} \mu_{\alpha, \beta}=0$, Proposition 1.9 implies:

$$
\int_{\partial \Gamma_{s}} f \mu_{\alpha, \beta}=0 \quad(\alpha, \beta=0,1,2, \ldots) .
$$

Therefore also for $n=2$ we have: $F_{h}^{s}(\zeta)=F_{h^{\prime}}^{s}(\zeta)$.

It remains to show that every $F_{h}^{s}$ is holomorphic, i.e. that, for each $\zeta \in U_{s}(\varphi) \backslash \partial D$,

$$
\frac{\partial F_{h}^{s}}{\partial \bar{\zeta}_{\alpha}}(\zeta)=0 \quad(\alpha=1, \ldots, n)
$$

Clearly, we have:

$$
\frac{\partial F_{h}^{s}}{\partial \bar{\zeta}_{\alpha}}(\zeta)=\int_{\Gamma_{s}} f \frac{\partial \omega}{\partial \bar{\zeta}_{\alpha}}(\zeta)-\int_{\partial \Gamma_{s}} f \frac{\partial \Phi_{h}}{\partial \bar{\zeta}_{\alpha}}(\zeta)
$$


further, on account of (1.5), (1.6), (1.7), we may rewrite the right side of this equality as:

$$
\int_{\Gamma_{s}} f \bar{\partial}\left[\frac{\partial \Omega_{\alpha}}{\partial \bar{\zeta}_{\alpha}}(\zeta)\right]-\int_{\partial \Gamma_{s}} f \frac{\partial \Omega_{\alpha}}{\partial \bar{\zeta}_{\alpha}}(\zeta)+I
$$

where

$$
I= \begin{cases}\int_{\partial \Gamma_{s}} f \bar{\partial} \Psi_{h}^{\alpha}(\zeta) & \text { if } n \geq 3 \\ 0 & \text { if } n=2 .\end{cases}
$$

Since $\left[\partial \Omega_{\alpha} / \partial \bar{\zeta}_{\alpha}\right](\zeta)$ is defined on all of $\mathbf{C}^{n} \backslash \zeta$, Proposition 1.9 implies that the difference of integrals in (*) is zero. Moreover, by Proposition 1.9 again, $I$ is zero also in case $n \geq 3$, since $\Psi_{h}^{\alpha}(\zeta)$ may be replaced by any $(n, n-3)$-form $\tilde{\Psi}^{\alpha}$ of class $C^{\infty}$ on all of $\mathbf{C}^{n}$ and equal to $\Psi_{h}^{\alpha}(\zeta)$ on an open neighbourhood of $\partial \Gamma_{s}$. Hence $\left[\partial F_{h}^{s} / \partial \bar{\zeta}_{\alpha}\right](\zeta)=0$.

The proof of Proposition 2.5 is then completed.

Next, we have:

Proposition 2.6. Let $V$ be an open neighbourhood of $\partial D \backslash K$, contained in $\bigcup_{U \supset \bar{D}} \bigcup_{\varphi \in \mathcal{O}(U)} U(\varphi)$, such that $V \backslash(\partial D \backslash K)=V_{+} \cup V_{-}$, where $V_{+}, V_{-}$are connected separated open sets and $V_{-} \subset \mathbf{C}^{n} \backslash \bar{D} .{ }^{8}$ Then $F=0$ on $V_{-}$.

Proof. We first point out that, given an open neighbourhood $U$ of $\bar{D}$ and a function $\varphi \in \mathcal{O}(U)$, if $\zeta$ is a point in $U$ such that $|\varphi(\zeta)|>\max _{\bar{D}}|\varphi|$ (which obviously implies that $\zeta \in U_{1}(\varphi) \backslash \bar{D}$ ), then $F(\zeta)=0$. As a matter of fact, if $h \in \mathcal{O}_{\varphi}^{n}(U \times U)$, we have:

$$
F(\zeta)=F_{h}^{1}(\zeta)=\int_{\Gamma_{1}} f \omega(\zeta)-\int_{\partial \Gamma_{1}} f \Phi_{h}(\zeta),
$$

and, since $\bar{D} \subset U \backslash L_{\zeta}(\varphi)$, on an open neighbourhood of $\bar{D} \omega(\zeta), \Phi_{h}(\zeta)$ are both defined and satisfy $\omega(\zeta)=\bar{\partial} \Phi_{h}(\zeta)$. Hence Proposition $1.9 \mathrm{im}$ plies that $F(\zeta)=0$.

Now, take $U$ and $\varphi$ such that $U(\varphi) \cap D \neq \varnothing$; then $\max _{\bar{D}}|\varphi|>$ $\max _{K}|\varphi|$, so that $\varphi$ is not constant on the connected component of $U$ containing $\bar{D}$ and, further, any point $\zeta^{0} \in \partial D$ where $|\varphi|$ attains the value

${ }^{8}$ Such a $V$ does exist, because $\partial D \backslash K$ is connected. For example, we may take as $V$ a small tubular neighbourhood of $\partial D \backslash K$ in $\mathbf{C}^{n} \backslash K$. 
$\max _{\bar{D}}|\varphi|$ must belong to $\partial D \backslash K$. One can actually find such a point $\zeta^{0}$ by the well known "maximum principle". Then $\zeta^{0}$ is a limit point of the open set $W=\left\{\zeta \in U ;|\varphi(\zeta)|>\max _{\bar{D}}|\varphi|\right\}$ (by the maximum principle again), and, since $\zeta^{0} \in \partial D \backslash K$, this obviously implies that $W \cap V_{-} \neq \varnothing$. But we already know that $F$ is zero on $W \cap V_{-}$; it follows that $F$ is zero on all of $V_{-}$, because $V_{-}$is connected.

Finally, we are in a position to prove that $F$ is a continuous extension of $f$ to $\bar{D} \backslash K$, i.e., the following holds:

Proposition 2.7. For every point $z^{0} \in \partial D \backslash K$ we have:

$$
\lim _{\zeta \rightarrow z^{0}} F(\zeta)=f\left(z^{0}\right)
$$

the limit being evaluated for $\zeta \in D$.

Proof. For every $w \in \partial D \backslash K$, denote by $\vec{\nu}(w)$ the unit vector perpendicular to $\partial D \backslash K$ at $w$, inward pointing with respect to $D$. We first prove that

$$
\lim _{t \rightarrow 0^{+}} F(w+t \vec{\nu}(w))=f(w)
$$

with the limit uniform on compact subsets of $\partial D \backslash K$. Given $w \in \partial D \backslash K$, we can find an open neighbourhood $U$ of $\bar{D}$, a function $\varphi \in \mathcal{O}(U)$ and a positive integer $s$ such that $w \in U_{s}(\varphi) \cap\left(\Gamma_{s} \backslash \partial \Gamma_{s}\right)$. Then, for $t>0$ small enough, we have:

$$
w+t \vec{\nu}(w) \in U_{s}(\varphi) \cap D, \quad w-t \vec{\nu}(w) \in U_{s}(\varphi) \cap V_{-},
$$

with $V_{-}$as in Proposition 2.6, and hence, if $h \in \mathcal{O}_{\varphi}^{n}(U \times U)$, it follows that

$$
\begin{aligned}
& F(w+t \vec{\nu}(w))=F_{h}^{s}(w+t \vec{\nu}(w)), \\
& F(w-t \vec{\nu}(w))=F_{h}^{s}(w-t \vec{\nu}(w))=0
\end{aligned}
$$

Therefore we may write:

$$
\begin{aligned}
F(w+t \vec{\nu}(w)) & =F_{h}^{s}(w+t \vec{\nu}(w))-F_{h}^{s}(w-t \vec{\nu}(w)) \\
& =I_{1}(w, t)-I_{2}(w, t)
\end{aligned}
$$

where

$$
\begin{aligned}
& I_{1}(w, t)=\int_{\Gamma_{s}} f[\omega(w+t \vec{\nu}(w))-\omega(w-t \vec{\nu}(w))] \\
& I_{2}(w, t)=\int_{\partial \Gamma_{s}} f\left[\Phi_{h}(w+t \vec{\nu}(w))-\Phi_{h}(w-t \vec{\nu}(w))\right] .
\end{aligned}
$$


Now, it can be shown that, for any $f \in C^{0}\left(\Gamma_{s}\right)$ (not necessarily a CRfunction) and $w \in \Gamma_{s} \backslash \partial \Gamma_{s}$,

$$
\lim _{t \rightarrow 0^{+}} I_{1}(w, t)=f(w),
$$

with the limit uniform on compact subsets of $\Gamma_{s} \backslash \partial \Gamma_{s}$. A similar result can be found in Harvey-Lawson [4], pp. 251-252, and the proof given there (based on a suitable estimate for $\|\omega(w+t \vec{\nu}(w))-\omega(w-t \vec{\nu}(w))\|)$ works essentially for the present case as well. ${ }^{9}$ Next, since the function $\zeta \mapsto \int_{\partial \Gamma_{s}} f \Phi_{h}(\zeta)$ is defined and real analytic on all of $U_{s}(\varphi)$, it is plain that, for $w \in U_{s}(\varphi) \cap\left(\Gamma_{s} \backslash \partial \Gamma_{s}\right)$,

$$
\lim _{t \rightarrow 0^{+}} I_{2}(w, t)=0,
$$

with the limit uniform on compact subsets of $U_{s}(\varphi) \cap\left(\Gamma_{s} \backslash \partial \Gamma_{s}\right)$. Hence (*) follows.

After that, it is easy to prove Proposition 2.7. Given $\varepsilon>0$, let $N_{z^{0}}$ be an open neighbourhood of $z^{0}$ in $\partial D \backslash K$ such that $\left|f(w)-f\left(z^{0}\right)\right|<\varepsilon / 2$, for every $w \in N_{z^{0}}$, and $N_{z^{0}} \Subset \partial D \backslash K$. Further, let $t_{0}>0$ be such that $|F(w+t \vec{\nu}(w))-f(w)|<\varepsilon / 2$, for every $t \leq t_{0}$ and $w \in \bar{N}_{z^{0}}$. Clearly, if $\zeta$ is a point of $D$ close enough to $z^{0}$, there exist exactly a point $w \in N_{z^{0}}$ and a positive number $t \leq t_{0}$ such that $\zeta=w+t \vec{\nu}(w)$. It follows that

$$
\left|F(\zeta)-f\left(z^{0}\right)\right| \leq|F(w+t \vec{\nu}(w))-f(w)|+\left|f(w)-f\left(z^{0}\right)\right|<\varepsilon,
$$

which proves Proposition 2.7.

Now the proof of Theorem 1 is completed.

\section{REFERENCES}

[1] G. Fichera, Teoria delle funzioni analitiche di più variabili complesse, Istituto Matematico "G. Castelnuovo", Univ. di Roma, notes, 1982-83.

[2] K. O. Friedrichs, The identity of weak and strong extensions of differential operators, Trans. Amer. Math. Soc., 55 (1944), 132-151.

[3] F. R. Harvey, Integral Formulae Connected by Dolbeault's Isomorphism, Rice Univ. Studies, 56 (1969), 77-97.

[4] F. R. Harvey and H. B. Lawson, On boundaries of complex analytic varieties, I, Ann. Math., 102 (1975), 223-290.

[5] L. Hörmander, An Introduction to Complex Analysis in Several Variables, North-Holland Publishing Company, 1973.

[6] G. Lupacciolu and G. Tomassini, Un teorema di estensione per le CR-funzioni, Ann. Mat. pura appl., 137 (1984), 257-263.

\footnotetext{
${ }^{9}$ The parallel result for $n=1$ and $\omega(\zeta)=(1 / 2 \pi i) \cdot d z /(z-\zeta)$ (the Cauchy kernel) goes back to Plemelj (cf. Muskhelishvili [9], pp. 43-45).
} 
[7] E. Martinelli, Sopra una dimostrazione di R. Fueter per un teorema di Hartogs, Comment. Math. Helv., 15 (1942), 340-349.

[8] J. R. Munkres, Elementary Differential Topology, Annals of Math. Studies 54, Princeton Univ. Press, Princeton, N. J., 1966.

[9] N.I. Muskhelishvili, Singular Integral Equations, Wolters-Noordhoff Publishing, Gronigen the Netherlands, 1958.

[10] G. Tomassini, Extension of CR-functions, to appear in Lecture Notes in Math., Springer-Verlag, New York.

[11] H. Whitney, Geometric Integration Theory, Princeton Univ. Press, Princeton, N. J., 1957.

[12] E. L. Stout, Analytic continuation and boundary continuity of functions of several complex variables, Proc. Edinburgh Royal Soc., 89A (1981), 63-74..$^{10}$

Received October 22, 1984.

istituto Matematico “Guido Castelnuovo"

UNIVERSITA DI ROMA "LA SAPIENZA"

00185 RoMA

${ }^{10}$ Added in proof. 



\section{PACIFIC JOURNAL OF MATHEMATICS EDITORS}

\author{
V. S. VARADARAJAN \\ (Managing Editor) \\ University of California \\ Los Angeles, CA 90024 \\ HeRbert Clemens \\ University of Utah \\ Salt Lake City, UT 84112 \\ R. FINN \\ Stanford University \\ Stanford, CA 94305
}

HERMANN FLASCHKA

University of Arizona

Tucson, AZ 85721

RAMESH A. GANGOLLI

University of Washington

Seattle, WA 98195

VAUGHAN F. R. JONES

University of California

Berkeley, CA 94720

ROBION KIRBY

University of California

Berkeley, CA 94720
C. C. MOORE

University of California

Berkeley, CA 94720

H. SAMELSON

Stanford University

Stanford, CA 94305

HAROLD STARK

University of California, San Diego

La Jolla, CA 92093

\section{ASSOCIATE EDITORS}
R. Arens
E. F. BECKENBACH
B. H. NEUMANN
F. WOLF
K. YoshIDA (1906-1982)

\section{SUPPORTING INSTITUTIONS}

UNIVERSITY OF ARIZONA

UNIVERSITY OF BRITISH COLUMBIA

CALIFORNIA INSTITUTE OF TECHNOLOGY

UNIVERSITY OF CALIFORNIA

MONTANA STATE UNIVERSITY

UNIVERSITY OF NEVADA, RENO

NEW MEXICO STATE UNIVERSITY

OREGON STATE UNIVERSITY
UNIVERSITY OF OREGON

UNIVERSITY OF SOUTHERN CALIFORNIA

TANFORD UNIVERSITY

UNIVERSITY OF HAWAII

UNIVERSITY OF TOKYO

UNIVERSITY OF UTAH

WASHINGTON STATE UNIVERSITY

UNIVERSITY OF WASHINGTON 


\section{Pacific Journal of Mathematics}

Vol. 124, No. $1 \quad$ May, 1986

Kinetsu Abe and Martin Andrew Magid, Relative nullity foliations and indefinite isometric immersions

Erik P. van den Ban, A convexity theorem for semisimple symmetric

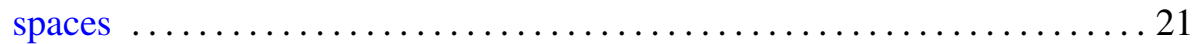

Bo Berndtsson and Thomas Joseph Ransford, Analytic multifunctions, the $\bar{\partial}$-equation, and a proof of the corona theorem .................. 57

Brian Boe and David H. Collingwood, Intertwining operators between

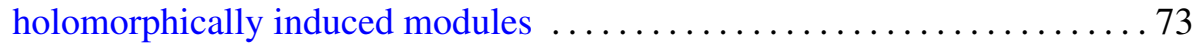

Giuseppe Ceresa and Alessandro Verra, The Abel-Jacobi isomorphism for

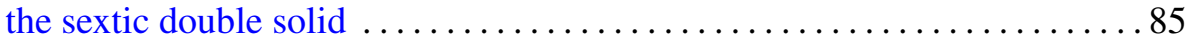

Kun Soo Chang, Jae Moon Ahn and Joo Sup Chang, An evaluation of the conditional Yeh-Wiener integral ........................... 107

Charles Dale Frohman, Minimal surfaces and Heegaard splittings of the

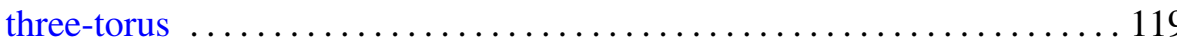

Robert M. Guralnick, Power cancellation of modules $\ldots \ldots \ldots \ldots \ldots \ldots 131$

Kenneth Hardy and Kenneth S. Williams, On the solvability of the Diophantine equation $d V^{2}-2 e V W-d W^{2}=1 \ldots \ldots \ldots \ldots \ldots \ldots \ldots \ldots \ldots$

Ray Alden Kunze and Stephen Scheinberg, Alternative algebras having

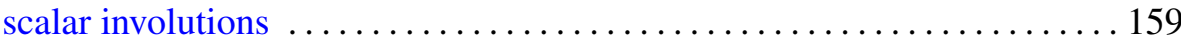

W. B. Raymond Lickorish and Kenneth Millett, The reversing result for the Jones polynomial $\ldots \ldots \ldots \ldots \ldots \ldots \ldots \ldots \ldots$

Guido Lupacciolu, A theorem on holomorphic extension of CR-functions

William Schumacher Massey and Lorenzo Traldi, On a conjecture of K. Murasugi

Dinakar Ramakrishnan, Spectral decomposition of $L^{2}(N \backslash \mathrm{GL}(2), \eta)$

Steven L. Sperber, On solutions of differential equations which satisfy certain algebraic relations 